REVISTA

de la

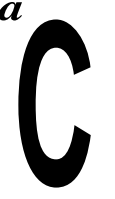

E

PAL

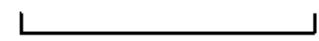

NUMERO 63

DICIEMBRE 1997

SANTIAGO DE CHILE

OSCAR ALTIMIR

Director

EUGENIO LAHERA

Secretario Técnico

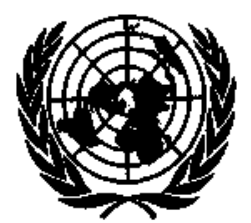

NACIONES UNIDAS 
El papel del sector público en el desarrollo latinoamericano

Ricardo Carciofi

La equidad en el presupuesto público

Juan Martin

Reformas a los sistemas de pensiones, mercado de capitales y ahorro

Andras Uthoff

Institucionalidad pública y políticas ambientales explícitas

e implícitas

Nicolo Gligo

La valoración de recursos naturales y ambientales no basada en el mercado en Centroamérica y el Caribe

Steve Shultz

Un modelo macroeconómico integrado para el Caribe

Lucio Vinhas de Souza

Virajes y derrapajes de la economía venezolana

José Miguel Benavente

¿Cuán no tradicionales son las exportaciones no tradicionales?

La experiencia de siete países de la Cuenca del Caribe

Alberto Gabriele

Apertura comercial y cambio estructural en la industria automotriz brasileña

Ruy de Quadros Carvalho, Sergio Robles Reis de Queiroz,

Flávia Luciane Consoni, loriara Costa y Janaína Pamplona da Costa

Historia evolutiva de una planta metalmecánica chilena

Jorge Katz y Héctor Vera

La importancia de la producción local y la pequeña empresa para el desarrollo de América Latina

Francisco Alburquerque

Publicaciones recientes de la CEPAL 


\section{El papel del sector público en el desarrollo latinoamericano}

\section{Ricardo Carciofi}

Banco Interamericano

de Desarrollo (BID),

Buenos Aires, Argentina
El papel del sector público en el desarrollo de los países latinoamericanos asiste a una transformación importante. El esquema de acción e intervención del Estado gestado en la posguerra culminó a comienzos de los ochenta, cuando la crisis de la deuda forzó el fin de un ciclo y el inicio de una readecuación a nuevas circunstancias. En este artículo se busca caracterizar las principales modificaciones de dicho papel y examinar las causas que determinaron el nuevo modelo de funcionamiento del sector público. Tanto durante el proceso de ajuste externo como en la fase posterior, cuando la región recobró su acceso al crédito, la disponibilidad y reacción de los mercados financieros fueron decisivas, Las conductas de los oferentes de fondos e inversores - internos y externos - han contribuido a delimitar el espacio para políticas que, tras diversos intentos y con modalidades que difieren de un país a otro, han alterado los mecanismos de la economía pública. El artículo concluye que los atributos básicos del nuevo esquema son los siguientes: el sector privado se constituye en el factor central dé movilización del ahorro y la inversión y la provisión de servicios sociales - aunque con distintas formas de gestión y financiamiento- queda en la órbita pública, con creciente descentralización de la tributación y el gasto público, y premisas diferentes para el diseño del sistema tributario. La configuración emergente no está aún definitivamente estructurada, pero las piezas que la conforman tienen un elementos de permanencia a mediano y largo plazo. De hecho, en el modelo de economía pública conocido hasta los años ochenta se ha operado una profunda transformación. 


\section{La conveniencia de una mirada retrospectiva}

Las funciones y el papel que desempeñó el sector público en el desarrollo de los países latinoamericanos, particularmente desde la posguerra hasta comienzos de los años ochenta, sufren hoy una transformación de vastas proporciones. Este cambio es fácilmente perceptible y se revela de manera casi cotidiana a través de los temas y noticias que nutren la discusión habitual sobre las políticas públicas en los diferentes países. Pero la magnitud de la transformación y lo simple que es comprobarla no eximen de la necesidad de realizar un esfuerzo de interpretación del fenómeno en curso. Un fenómeno que más allá de la globalización de las tendencias económicas, que apunta a eliminar los elementos propios y las especificidades de los países, tiene una manifestación particular en el caso de América Latina.

Con el propósito de apreciar la naturaleza de las transformaciones actuales del papel de la economía pública en la región, parece aconsejable partir con una mirada retrospectiva, no para hacer historia, sino simplemente para caracterizar las diferencias e intentar además una explicación de lo que ha llevado a modificar las funciones del sector público en las economías latinoamericanas.

\section{II}

\section{El sector público: la movilización de ahorro e inversión y el crecimiento}

De partida, y como referencia para el análisis, es útil recordar la taxonomía de Musgrave sobre las funciones del sector público: asignación, distribución y estabilización. Estas tres categorías son utilizadas de manera habitual para justificar desde el ángulo económico la absorción de recursos y su aplicación a través del gasto público. Al confrontar estas tres dimensiones, cabe preguntarse cuál de ellas parece haber predominado en la inspiración de las orientaciones y políticas que moldearon los sectores públicos de la región desde la posguerra en adelante. La hipótesis que adelantamos aquí es que ninguna de las tres ofrece una explicación satisfactoria. Es más, podría afirmarse que el esquema que subyace la presentación de Musgrave no es el más apropiado a la hora de un análisis en perspectiva histórica.

La concepción que fue dando forma a la acción y dimensión de los sectores públicos de América Latina tuvo un punto de arranque distinto: el desafío fue, inva-

\footnotetext{
8 ' Las opiniones vertidas en este trabajo son de carácter personal y no reflejan necesariamente las de la institución a la que pertenece el autor.
}

riablemente, la búsqueda del desarrollo y la aceleración del proceso de crecimiento e industrialización. Para alcanzar estos objetivos el flanco débil era la fragilidad externa de las economías, que se constituyó en la preocupación dominante en la política económica de la región a partir de los años treinta. En esta dependencia externa influían múltiples factores: los ciclos de financiamiento, los vaivenes de los precios y la demanda asociados a la exportación de recursos naturales, el rezago de la explotación agropecuaria — donde la estructura de la tenencia de la tierra propiciaba una conducta rentística- y el drenaje de recursos originado por la transferencia de utilidades al exterior. Inscrito en este cuadro, el sector público fue adquiriendo un papel cada vez más importante en la movilización de ahorro, destinado por una parte a la formación de capital básico en estas economías, y por otro, a una provisión más amplia de servicios sociales. Vemos así que, en contraste con las categorías de Musgrave, estas funciones eran considerablemente más vastas y se las concebía de manera dinámica. Los instrumentos de la política fiscal, que combinaban formas e intensidades diversas, iban más allá de la simple corrección de fallas de mercado, o la aspiración de subsanar 
inequidades distributivas mediante el suministro de bienes meritorios o a través de impuestos y pagos de transferencias. Como se dijo arriba, el vigor impreso al sector público latinoamericano tenía como telón de fondo el desafío de promover el crecimiento frente a mercados cuyas fallas no se enraizaban en la tipología tradicional, sino más bien en las propiedades del subdesarrollo.

Esta tarea del sector público de movilizar ahorro y ser principal motor de la inversión se apoyó en diversos instrumentos que la hicieron posible. Así, la generación de superávit públicos se gestó utilizando fuentes que probaron ser efectivas: la captación de rentas monopólicas, canalizadas por lo general a través de las empresas estatales dedicadas a la explotación de recursos naturales; el uso del ahorro corriente generado por la producción de aquellos servicios públicos (la electricidad, el transporte ferroviario, etc.) que seguían aprovechando las inversiones iniciales, generalmente extranjeras, y que todavía no demandaban grandes ampliaciones o renovaciones; los fondos de la seguridad social - en materia de pensiones y seguros de saludque se beneficiaban financieramente de la expansión horizontal y la incorporación continua de nuevos afiliados; y finalmente también, la captación de ahorro externo en años como los cincuenta y los sesenta, en que los pocos recursos disponibles para la región llegaban a través de fuentes oficiales.

Por su parte, los recursos captados se canalizaban hacia diferentes destinos: a la ampliación significativa de diversos programas sociales en áreas como salud, vivienda y educación media y superior; a la formación de empresas en diversos sectores industriales, o a la participación mayoritaria en paquetes accionarios en ramas que, con criterios no siempre coincidentes, se juzgaban de importancia clave (siderurgia, petroquímica, cemento, etc). Asimismo, en muchos casos las nuevas empresas públicas o entes gubernamentales tuvieron como responsabilidad directa la promoción del desarrollo de regiones enteras y la construcción de nueva infraestructura (comunicaciones, red vial troncal, generación de energía, etc). También los recursos públicos actuaron en auxilio de la inversión privada: de manera directa a través del sistema de promoción industrial e indirecta a través de los precios relativos, mediante el sistema financiero y la protección arancelaria.

La estrategia aplicada, a juzgar por los trazos centrales comentados y por su trayectoria desde la posguerra hasta fines de los años setenta, arrojó resultados nada despreciables. El sector público se convirtió paulatinamente en un factor importante de acumulación de capital, contribuyó al desarrollo industrial y promovió la creación de una red de servicios sociales suficientemente amplia. Por cierto, caben las salvedades de rigor en cuanto a las pronunciadas diferencias entre los diversos países de la región. Sin duda, la descripción trazada se ajusta mejor a las economías latinoamericanas de mayor tamaño, donde las políticas de industrialización alcanzaron mayor profundidad.

\section{III}

\section{Elementos de tensión: las restricciones}

Sin embargo, junto con señalar la trayectoria reseñada, corresponde destacar algunos atributos que, juzgados ahora con perspectiva histórica, emergen como elementos de tensión creciente que se convertirían en restricciones críticas en los años ochenta.

En primer lugar, parece haberse dado poca importancia al papel de la política fiscal como instrumento anticíclico. Las variables fiscales no se concibieron, en general, con el grado de flexibilidad y los márgenes de maniobra apropiados para actuar como correctivo frente al ciclo económico. En parte, esto se explica por las circunstancias y la forma de gestación de los ciclos: en la mayoría de los países éstos se asociaban a impactos de origen externo, lo que reducía las posibilidades de contrarrestarlos con instrumentos fiscales. En todo caso, el papel asignado al sector público en el proceso de ahorro e inversión hizo que frecuentemente se olvidara el conjunto de restricciones macroconómicas al que aquél debía ajustarse. En parte, el claro síndrome inflacionario que sufrieron varias de las economías latinoamericanas en aquellos años se asocia a la laxitud de la política fiscal. Tal laxitud, a su vez, se originaba en las demandas concurrentes - y excesivas - que pesaban de manera simultánea sobre el sector público. Sin embargo, la imposibilidad de atender estas demandas, con la correspondiente aparición de desequi- 
librios fiscales de magnitud, no fue una tónica recurrente ni uniforme. No es casual que los déficit fiscales se hayan visto agravados a mitad del decenio de 1970. En los años previos a este período existió cierto equilibrio entre el costo de atender a las múltiples funciones que abarcaba el presupuesto gubernamental, y la disponibilidad de diversas rentas estatales. Cuando el equilibrio se quebró de uno u otro lado, el sector público cayó en desequilibrios financieros. En algunos países éstos dieron lugar a severas dificultades para la política macroeconómica de corto plazo, que condujeron en última instancia a episodios inflacionarios.

En segundo lugar, se tendió a otorgar escasa importancia a las distorsiones en la asignación de recursos asociadas a las formas de intervención estatal. Los ejemplos son múltiples: el sistema impositivo persiguió diversos objetivos contrapuestos aun en situaciones de administración tributaria deficiente (promoción de la industria, incentivos al autofinanciamiento de las empresas, complejidades de dudosa eficacia en la tributación sobre el consumo, etc); hubo abuso de los subsidios cruzados en las tarifas y precios de los servicios públicos y se evaporaron recursos financieros que debían constituir los fondos de reserva para garantizar los derechos que otorgaba el propio sector público (las pensiones son el ejemplo más claro).

En tercer término, todo el modelo de intervención pública se desarrolló bajo una tónica fuertemente centralista, aun en los países que tenían instituciones de corte federativo. Nuevamente se pueden identificar aquí factores de diverso orden que ayudan a explicar estas tendencias. Sin lugar a dudas, la forma de ocupación del territorio y la herencia institucional sobre la que se construye el Estado nacional fueron elementos importantes. Pero también favoreció el centralismo la presencia recurrente de gobiernos militares que, por imperio de su propias concepciones políticas y las circunstancias en las que actuaron, tendieron a obstaculizar formas institucionales y de participación democrática a nivel local.

Por último -aunque la enumeración podría extenderse-, la acumulación de capital público y las diversas formas de intervención involucraron pérdidas de productividad, principalmente por deficiencias de la propia gestión estatal. Pero estas pérdidas no eran vistas como costos evitables: en muchos casos el sector público era el único actor y partícipe de la gestión en muchas áreas y sectores de la producción de bienes y servicios. Faltaba un sector privado que sirviera de parámetro y comparación para la economía del sector público, y que actuara como inductor de competitividad y eficiencia. Pero había otra razón que ubicaba en segundo plano las eventuales pérdidas de productividad: los recursos de ahorro estaban efectivamente disponibles y los límites que imponía el presupuesto agregado del sector público no ponían en riesgo el conjunto de la estrategia. En pocas palabras, junto con haber progresos en la ampliación de la esfera de explotación estatal, se producían pérdidas en la asignación de recursos que no constituían motivo de preocupación para la política económica.

\section{IV}

\section{La crisis de 1982. Fin de un ciclo y readecuación a las nuevas circunstancias}

El modelo descrito dejó de aplicarse en la región, casi sin excepciones, en 1982. El final fue abrupto y estuvo asociado a la crisis de pagos externos que comenzó en México y que habría de extenderse posteriormente al resto de la región. Como se sabe, el caso singular fue Chile, cuya trayectoria se ajustó sólo parcialmente a la situación reseñada. Como el resto de América Latina, Chile acusó el impacto real y financiero de la crisis de la deuda; pero en este país las reformas económicas, en especial las vinculadas al papel del Esta- do, se habían iniciado ya en la segunda mitad de los años setenta. ${ }^{1}$

Si bien los acontecimientos que se desencadenaron a partir de 1982 pusieron un repentino punto final

\footnotetext{
${ }^{1}$ Las experiencias de otros países del Cono Sur en esos años no tienen la misma trascendencia para el examen que se ha hecho aquí sobre las funciones económicas del sector público, porque si bien se concentraron en reformas comerciales y financieras, no alteraron los trazos centrales de la economía pública.
} 
a las funciones que había cumplido hasta entonces el sector público en el proceso de desarrollo económico y social de los países de la región, esta transformación no fue un episodio instantáneo. Como veremos más adelante, los nuevos elementos que constituyen el modelo emergente se han ido plasmando de manera paulatina a partir de entonces, con ritmos y modalidades que difieren de un país a otro. Antes de referirnos a esta nueva configuración, parece útil subrayar tres aspectos que hacen a las circunstancias y factores que empujaron hacia el cambio.

En primer lugar, debe repararse en que, reiterando el ciclo previo de los años treinta, el impacto de perturbaciones externas fue el disparador de la transformación. Esta afirmación no conlleva una intención contrafactual. Es imposible aventurar cómo habría reaccionado el modelo vigente hasta entonces si no se hubiera producido la crisis de la deuda. Como ya se dijo antes, el desempeño del sector público venía acumulando dificultades en el plano financiero, en su encuadre macroeconómico y en aspectos de carácter sectorial en varios países de la región. No cabe descartar que estas dimensiones conflictivas podrían haber llevado a la aplicación de políticas correctivas. Sin embargo, esta es una conjetura que no se presta al análisis y los hechos conocidos indican que lo que impulsó la movilización y el cambio no fue la conciencia respecto de los problemas que venía acumulando el sector público, sino una modificación sustancial de las corrientes financieras externas y la necesidad de una rápida readecuación a las nuevas circunstancias.

En segundo lugar, llama la atención, al menos en un primer examen, que las políticas que siguieron los países latinoamericanos con posterioridad a la crisis de la deuda tuvieron un curso más o menos paralelo a los cambios que se daban en el mundo desarrollado. En la primera mitad de los años ochenta cobraron vigor las orientaciones de corte neoliberal, particularmente en Estados Unidos y Gran Bretaña. Bajo su amparo se concibieron y aplicaron medidas radicales en diferentes órbitas de la gestión económica. El aumento de la inflación, la recesión y el desempleo que siguió a las dos andanadas de alzas en los precios del petróleo en los años setenta dibujaron el escenario básico en que se produjo esta revisión del modelo aplicado hasta entonces. Se quebró así una tendencia que venía desde la posguerra, caracterizada por un acople de instrumentos keynesianos de manejo de la demanda agregada junto al desarrollo del Estado de bienestar. La concepción del gasto y la tributación —en su diseño ma- croeconómico y microeconómico - reflejaba ese modelo.

El cambio de orientación que se produjo en los países desarrollados estuvo acompañado por un debate sobre la eficacia, el costo económico y las consecuencias sociales del modelo seguido hasta entonces. No es de extrañar que en el nuevo contexto, donde los datos de la realidad no se asimilaban fácilmente a las premisas y resultados del análisis económico, se haya propuesto un cambio de fondo en el papel del sector público. El debate invadió y avivó los medios académicos, pero además los trascendió, al definir con fuerza el meollo de la discusión política.

Por la severidad y magnitud con que se hizo presente la crisis en América Latina, la secuencia de acontecimientos fue muy diferente de la observada en los países desarrollados. El debate fue más pragmático y con menos posibilidades de gestar una discusión profunda sobre las limitaciones del camino seguido hasta entonces y los nuevos fundamentos conceptuales de la transformación que habría de emprenderse. De manera bastante abrupta, las cuentas fiscales, que mostraban desequilibrios importantes al comenzar los años ochenta, enfrentaron restricciones de crédito. Las políticas debieron hacerse cargo de las nuevas condiciones de financiamiento externo, lo que requería una adaptación del gasto interno y la absorción interna de los bienes y servicios a la nueva situación. Por esta razón, las políticas estuvieron dominadas por las prioridades macroeconómicas asociadas al ajuste y la estabilización, y sus instrumentos se concibieron y ordenaron en función de estos objetivos. El análisis de las cuestiones de fondo quedó de cierta manera inhibido, puesto que el criterio de validación de las soluciones en juego era diferente. Los países latinoamericanos procuraron capear el temporal buscando readecuar las condiciones económicas internas a las condiciones del financiamento externo. Por esta razón varias de las experiencias de política económica heterodoxa observadas en la región durante aquellos años presentan pocas diferencias, sobre todo en los instrumentos de política fiscal utilizados, con los planteamientos que tradicionalmente caracterizaron a las inspiraciones neoliberales, pues coincidían en la necesidad de ubicar el desequilibrio fiscal a un nivel coherente con el resto de las restricciones presupuestarias agregadas. Las diferencias se referían a la intensidad y velocidad con que debían aplicarse las medidas de ajuste, o a la eventual intolerancia social ante dicho proceso.

En tercer lugar, cabe destacar una implicación natural de lo expuesto más atrás y que se refiere a los 
alcances macroeconómicos del proceso. En la medida en que la deuda externa fue contraída por el sector público o incorporada a los presupuestos públicos, las políticas fiscales no sólo debieron actuar como instrumento de equilibrio interno y estabilización. Como se sabe, dichas políticas fueron una herramienta primordial para reducir el gasto agregado y por esta vía permitieron ampliar el excedente comercial externo. Y junto con cumplir esta función, los instrumentos fiscales debieron conducir la transferencia interna de ingreso que el proceso de ajuste requería. Por las magnitudes en juego, y dependiendo de las situaciones particulares que enfrentaban las distintas economías al inicio de la crisis, esta redistribución de ingresos y activos fue un proceso complejo, que en varios países adquirió características muy conflictivas: inflación alta, estancamiento, destrucción de los mercados financieros internos y predominio de un clima de incertidumbre económica.

Ya se ha afirmado que la duración del ambiente de crisis y la severidad de ella no fueron uniformes en la región. Hubo países como Costa Rica y Chile que contaron con mayor financiamiento externo en períodos críticos, y otros que no habían incurrido en endeudamiento desmedido, como Colombia. En cambio, Argentina, Brasil y México atravesaron circunstancias más difíciles. Por esta razón, el proceso de ajuste se caracterizó por episodios de desigual duración. Con independencia de estas disparidades y de las diversas reacciones de las políticas nacionales al nuevo contexto, los sectores públicos de la región fueron manifestando, ahora de manera explícita, la fase de agotamiento en la que se encontraban. Los síntomas no se develaron en aspectos sectoriales o relativamente acotados de las políticas de gasto o tributación. Es cierto que la inversión en infraestructura pública, los sistemas de pensiones, los servicios sociales y los sistemas impositivos fueron sacudidos por la crisis y mostraron sus limitaciones. Sin embargo, un elemento que coordinó la reacción y que ha constituido un factor importante de cambio, especialmente a partir de los años noventa, fue la pérdida de la reputación crediticia del sector público.

Esta pérdida debe ser interpretada de manera amplia, porque se manifiesta tanto en un uso muy acotado de la facultad de cobrar el impuesto inflacionario, como en la posibilidad de recurrir sólo dentro de límites muy estrechos a los mercados de crédito. En este sentido podría hablarse de una cierta simetría entre el punto de origen y la continuidad posterior del proceso. La perturbación inicial que conmovió las funciones económicas del sector público latinoamericano fue de carácter macroeconómico. En los años recientes, y a pesar de haberse reconstituido los flujos de financiamiento externo hacia la región, los mercados de crédito - tanto locales como internacionales- señalan un claro límite a las presiones que puede ejercer sobre ellos el sector público. Esta restricción, de carácter agregado, ha sido el factor dominante que ha moldeado y definido el espacio de las políticas de tributación y gasto público.

Así, los sectores públicos no sólo están obligados a conservar el balance fiscal o preservar un modesto desequilibrio. Aun adoptando posiciones financieramente prudentes, las políticas fiscales deben ofrecer ingredientes estructurales que señalen de forma inequívoca que apuntan a una redefinición profunda del papel económico del Estado. Sería erróneo, siguiendo la interpretación esbozada, suponer que estas demandas de cambio resultan tan sólo de nuevas orientaciones y condicionalidades de los organismos internacionales o, más vagamente, de factores externos. El núcleo de este proceso es una modificación radical en las formas de funcionamiento de los mercados financieros. En cierta manera las políticas públicas en general, pero muy especialmente las que hacen al sistema fiscal, tienen radios de maniobra delimitados por la proclividad de los mercados financieros a aceptar el contenido de esas políticas. Los mercados han mostrado su disposición a facilitar crédito al sector privado aun aceptando los riesgos del desequilibrio en las cuentas comerciales externas, con condición explícita de que los países emprendieran reformas en diversos campos, en especial vinculadas al sector público. Las conductas de endeudamiento e inversión del sector privado interno también se han movido bajo premisas similares. Como es obvio, esta forma de operación de los mercados -incluidas las reacciones de éstos, el procesamiento de la información que reciben, etc. - ha cobrado un relieve cada vez mayor a partir de la globalización de las corrientes financieras. Y también la ha favorecido desde tiempo atrás la sustitución de los títulos de deuda por activos como resultado del proceso de privatización. 
V

\section{Los elementos constitutivos del}

\section{modelo emergente}

Como vimos en la sección anterior, una buena parte de los años ochenta estuvo animada por la necesidad de llevar a cabo el proceso de ajuste; las reformas estructurales, de la gama y variedad que se vienen aplicando hoy en la mayor parte de los países de la región, debieron aguardar mejores tiempos. El vuelco favorable de la situación se ha apoyado en diversos elementos. Los cambios en los arreglos institucionales sobre la renegociación de la deuda a largo plazo han significado un progreso. Los ha seguido el apoyo de los organismos multilaterales de crédito. También ha sido un factor positivo la reanudación de la afluencia de capitales hacia la región que comenzó en 1990 y que se ha mantenido hasta la fecha, no obstante los episodios de fines de 1994 y 1995 que afectaron particularmente a la economía mexicana y argentina. Este masivo acceso de América Latina al crédito internacional permitió la recuperación y el crecimiento económicos, aunque también puso de manifiesto nuevamente la frágil situación en la que se encontraban varias economías de la región. En adelante las políticas macroeconómicas nacionales deberán adecuarse, con los instrumentos a su alcance, al nuevo marco. La disponibilidad de financiamiento externo y la posibilidad de que se reviertan parcialmente las tendencias recientes habrán de condicionar la evolución económica en el futuro inmediato.

Cualesquiera sean las alternativas que deparen estos nuevos acontecimientos en el futuro, entendemos que la interpretación esbozada en el punto anterior sigue siendo válida y que, desde ese ángulo, no es dable esperar una reversión del camino seguido. La restricción al crédito público sigue siendo dominante, y ha tenido algunos efectos que se harán sentir a mediano y largo plazo. Las reformas estructurales ya se han aplicado en varias de las órbitas de la economía pública - privatizaciones de los servicios e infraestructura pública, rediseño de los sistemas de pensiones, reformas de los sistemas tributarios, etc.-, e indican una desviación apreciable respecto de la tendencia conocida. Además, las nuevas piezas del esquema no son, en términos económicos y políticos, de fácil sustitución; siendo así, parece probable que los países que han iniciado este proceso continuarán profundizándolo. Otros, como Brasil, cuyas reformas estuvieron inicialmente a la zaga de las del resto de la región, en la actualidad ofrecen indicios firmes de avanzar en la misma dirección.

En síntesis, el sector público de la mayor parte de los países de la región está asistiendo a una profunda redefinición de sus funciones. La amplitud y diversidad de las reformas en curso vinculadas de manera esencial al desempeño de estas funciones varían ampliamente en el concierto de la región. Aunque apuntan en direcciones similares, esas reformas exhiben importantes diferencias instrumentales que influyen en sus efectos económicos y sociales. Sin embargo, lo que interesa destacar aquí son los elementos que imprimen una nota común y que, por lo tanto, permiten entrever que nos encontramos ante un cambio de modelo que divide las aguas respecto del pasado. Entendemos que los atributos centrales del nuevo modelo son los que se enumeran a continuación.

\section{Redefinición del papel del sector público en el proceso de ahorro e inversión}

El sector público, que había sido un factor central en la movilización del ahorro y la inversión, ve emerger ahora un nuevo actor: el sector privado. Una porción apreciable de las actividades económicas con mayor rentabilidad y potencial de crecimiento ha sido transferida al capital privado para su explotación. Estas fuentes de rentas forman parte ahora del patrimonio privado. Además, particularmente en aquellos países donde los cambios de propiedad han incluido importantes áreas de infraestructura que tradicionalmente estaban bajo control estatal — como la red vial y ferroviaria, los puertos, la energía eléctrica, el agua potable y el saneamiento, las comunicaciones, etc.también son de resorte privado la decisión y la gestión de inversiones en ellas. Del mismo modo, se han transferido recursos naturales y se han viabilizado regímenes que permiten efectuar importantes inversiones privadas en este campo. Ya no están abiertos los viejos mecanismos de transferencia de ingresos que fueron posibles en su momento gracias a la protección arancelaria, el crédito subsidiado o la captación de excedentes financieros. Un ejemplo claro de esto último es la aparición de los fondos de pensiones en virtud de la 
reforma de los sistemas previsionales: el uso de estos recursos en inversiones de largo plazo representa un nuevo espacio para las decisiones privadas en un terreno que antes les había estado tradicionalmente vedado.

Lo anterior no significa por cierto que frente a estas nuevas fuentes de ahorro y estos nuevos usos de los recursos habrá una total prescindencia del sector público. Pero el papel de este último cambia diametralmente: la gestión y decisión directas son sustituidas por la regulación y la aplicación de instrumentos indirectos. Esto exige nuevas capacidades estatales de planeación y administración, que están comenzando a construirse. El proceso no es instantáneo; en él se avanza más bien por aproximaciones sucesivas, y deberá ser considerado escenario natural para el diseño y la ejecución de las políticas públicas en estos nuevos ámbitos que ha abierto la reforma de la economía del sector público.

\section{Mayor concentración del sector público en la provisión de servicios sociales}

Habiendo transferido las actividades asociadas a la producción de bienes y servicios públicos e infraestructura, el sector público tiende a concentrarse mucho más en la provisión de servicios sociales. Pero las reformas de los mecanismos de provisión y financiamiento de esos servicios no han mostrado un dinamismo equivalente, aunque los problemas que acumulan los países de la región son de larga data y se han visto agravados por el impacto de las transformaciones recientes, en particular las asociadas a la restructuración de las actividades productivas y el mercado de trabajo en economías que han acrecentado su apertura.

Salvo en el caso de los sistemas de pensiones, las modalidades de intervención del sector público se dan en la actualidad bajo patrones ya conocidos. Esto, por cierto, no significa una ausencia de innovaciones. Observando con detalle la experiencia reciente de los países de la región, quedan de manifiesto políticas sectoriales —educación, salud, vivienda, programas de combate a la pobreza- que intentan subsanar algunas de las dificultades más notorias e innovar en otras materias. Sin embargo, los resultados efectivos de los programas públicos vinculados a la reforma social parecen estar demorados, en parte porque se tropieza con obstáculos más complejos, y el diseño de nuevos instrumentos de acción y financiamiento requiere un cambio de modalidades de organización, de estructuras administrativas y de recursos humanos dentro de la propia esfera del sector público. De por sí, todo esto supone cambios más lentos.

\section{Tendencia a la descentralización}

Otra razón que según creemos explica el menor avance registrado en este frente es la tendencia a la descentralización. Así como afirmamos que un elemento funcional del anterior modelo de funcionamiento del sector público fue su marcado centralismo, la actual etapa está marcada por una tendencia en el sentido opuesto. La descentralización de las actividades y de la gestión gubernamental tiene su claro correlato en la división de competencias dentro de las estructuras de las finanzas públicas nacionales. El proceso se caracteriza por la entrega a los gobiernos locales de mayores responsabilidades en la provisión de servicios públicos, particularmente los de carácter social.

En cuanto a los recursos tributarios y financieros que apoyan esta nueva división de facultades, las líneas de conducta que parecen poner de manifiesto los países de la región son menos nítidas. En algunos casos se promueven políticas de fortalecimiento de la capacidad de tributación local, que tropiezan con las dificultades conocidas: en los aspectos económicos, las estrechas o muy desiguales bases tributarias de las regiones, provincias o comunas, y en la dimensión política e institucional, la resistencia a asumir los costos políticos de los mayores impuestos o el enfrentamiento con poderes económicos locales. Por ello, en la mayoría de los países la descentralización parece estar acompañada de una reformulación de los sistemas de transferencias financieras que conectan a los distintos niveles de gobierno. Pero, de una manera u otra, el proceso de redespliegue geográfico del sector público muestra, quizás más que en el pasado, una dimensión regional en la puja por la distribución de las rentas y gastos públicos. Si bien lo que se ha reseñado aquí son los aspectos fiscales asociados a la descentralización, cabe agregar que la dinámica del proceso descentralizador depende estrechamente de factores cuya lógica y ritmo no siempre coinciden con los de ella. Los elementos principales que alimentan el proceso son de carácter político e institucional, y asumen modalidades propias según las diferentes realidades nacionales. De allí que si bien la dirección general del proceso se encamina hacia una mayor injerencia de los gobiernos subnacionales, las formas e instrumentos de esa injerencia no se prestan fácilmente a la generalización.

\section{Papel de la política tributaria}

En este campo, las reformas recientes aplicadas en los países de la región señalarían un cambio apreciable de objetivos. Como se sabe, hasta hace poco los sistemas 
tributarios prevalecientes en la región tenían incorporados — al menos en su diseño normativo - ambiciosos objetivos de redistribución de ingresos combinados con no menores pretensiones en la promoción de diferentes actividades productivas o regiones. Las nuevas políticas se han distanciado de estos objetivos. Hay una mayor renuencia a conceder beneficios tributarios particulares y se hace más hincapié en la equidad horizontal, a lo que se han sumado reglas más simples y universales para la tributación sobre el consumo. Por un lado, estos cambios indicarían un abandono de objetivos de progresividad tributaria y de la generación de estímulos indirectos para aumentar la formación de capital. Por otro lado, también puede juzgarse que el perfil distributivo tradicionalmente desalentador de América Latina sólo puede ser corregido marginalmente con una tributación más progresiva sobre la renta. Asimismo, se observa una menor propensión a aplicar desgravaciones y exenciones impositivas, debido a un balance más ponderado del costo-beneficio de los mecanismos que se aplicaban en el pasado. Las tendencias de las políticas tributarias actuales en la región, por lo tanto, parecen acentuar los objetivos recaudatorios, de simplificación y de equidad horizontal, combinados con la ampliación de las bases para los impuestos al consumo.

\section{VI}

\section{A modo de conclusión}

En la interpretación expuesta se reconocen tres argumentos esenciales. En primer lugar, hemos expuesto que el modelo de funcionamiento y el papel del sector público experimentaron cambios sustanciales tras la crisis de la deuda en los años ochenta. En segundo lugar, hemos dicho que tanto durante el proceso de ajuste externo como en la fase posterior en que la región recobró su acceso al crédito, fueron decisivas la acogida y la reacción de los mercados financieros a la orientación y contenido de las políticas de reforma en el sector público. Los comportamientos de los oferentes de fondos e inversores - internos y externos - contribuyeron a delimitar el espacio para políticas que, tras diversos intentos y con diferentes modalidades en los distintos países, han alterado los mecanismos de la economía pública. En tercer lugar, hemos afirmado que los atributos básicos del nuevo esquema son los si- guientes: redefinición del papel del Estado en el proceso de ahorro e inversión, concentración del sector público en la provisión y financiamiento del gasto social, descentralización y nuevas premisas para el diseño tributario.

La configuración que surgirá de estos elementos no está definitivamente plasmada: el proceso está aún incompleto y en desarrollo. Sin embargo, sus piezas tienen un elemento de permanencia a mediano y largo plazo. Cabe suponer, entonces, que lo que buscarán las políticas será resolver deficiencias y proponer correcciones en un curso de acción que, en sus aspectos esenciales, no parece estar sujeto a mayores reversiones. Podría afirmarse entonces que, en los hechos, el modelo de economía pública aplicado hasta los años ochenta ha experimentado una profunda transformación. 